\title{
The bright optical/NIR afterglow of the faint GRB 080710 - evidence of a jet viewed off-axis ${ }^{\star}$
}

\author{
${ }^{1}$ Max-Planck-Institut für extraterrestrische Physik, Giessenbachstrasse, 85748 Garching, Germany \\ e-mail: kruehler@mpe.mpg.de \\ 2 Universe Cluster, Technische Universität München, Boltzmannstrasse 2, 85748 Garching, Germany \\ 3 Thüringer Landessternwarte Tautenburg, Sternwarte 5, 07778 Tautenburg, Germany \\ 4 School of Physics, University College Dublin, Dublin 4, Ireland \\ 5 European Southern Observatory, 85748 Garching, Germany \\ ${ }^{6}$ Center for Astrophysics and Cosmology, University of Iceland, Dunhagi 5, 107 Reykjavík, Iceland \\ Institute of Physics, Eötvös University, Pázmány P. s. 1/A, 1117 Budapest, Hungary \\ 8 Department of Physics and Astronomy, Clemson University, Clemson, SC 29634, USA
}

T. Krühler ${ }^{1,2}$, J. Greiner ${ }^{1}$, P. Afonso ${ }^{1}$, D. Burlon ${ }^{1}$, C. Clemens ${ }^{1}$, R. Filgas ${ }^{1}$, D. A. Kann ${ }^{3}$, S. Klose ${ }^{3}$, A. Küpcü Yoldaş ${ }^{5}$, S. McBreen ${ }^{4}$, F. Olivares ${ }^{1}$, A. Rau ${ }^{1}$, A. Rossi ${ }^{3}$, S. Schulze ${ }^{3,6}$, G. P. Szokoly ${ }^{7}$, A. Updike ${ }^{8}$, and A. Yoldaş ${ }^{1}$

Received 7 June 2009 / Accepted 21 July 2009

\section{ABSTRACT}

\begin{abstract}
Aims. We investigate the optical/near-infrared light curve of the afterglow of GRB 080710 in the context of rising afterglows. Methods. Optical and near-infrared photometry was performed using the seven-channel imager GROND and the Tautenburg Schmidt telescope. X-ray data were provided by the X-ray Telescope onboard the Swift satellite. We construct an empirical light curve model using the available broadband data, which is well-sampled in the time and frequency domains.

Results. The optical/NIR light curve of the afterglow of GRB 080710 is dominated by an initial increase in brightness, which smoothly turns over into a shallow power law decay. At around $10 \mathrm{ks}$ post-burst, there is an achromatic break from shallow to steeper decline in the afterglow light curve with a change in the power law index of $\Delta \alpha \sim 0.9$.

Conclusions. The initially rising achromatic light curve of the afterglow of GRB 080710 can be accounted for with a model of a burst viewed off-axis or a single jet in its pre-deceleration phase and in an on-axis geometry. A unified picture of the afterglow light curve and prompt emission properties can be obtained with an off-axis geometry, suggesting that late and shallow rising optical light curves of GRB afterglows might be produced by geometric effects.
\end{abstract}

Key words. gamma rays: bursts - techniques: photometric

\section{Introduction}

The launch of the Swift satellite (Gehrels et al. 2004) in 2004 opened a new field of gamma-ray burst (GRB) afterglow physics. With its precise localization by the Burst Alert Telescope (BAT; Barthelmy et al. 2005), rapid slewing capabilities and early follow-up with two instruments in the X-ray and ultraviolet/optical regime, studies of the early afterglow phase were possible for the first time with larger sample statistics of around 100 per year.

Long GRBs are generally classified according to the spectral properties of their prompt emission. While conventional GRBs (CGRBs) have the peak energy of their observed spectrum in the $300 \mathrm{keV}$ range (Preece et al. 2000), the spectrum of X-ray rich bursts (XRRs) and X-ray flashes (XRFs) peak at significantly lower energies, typically around $50 \mathrm{keV}$ for XRRs or $10 \mathrm{keV}$ for XRFs respectively (e.g., Heise et al. 2001; Kippen et al. 2003). The spectral and temporal properties (e.g., Sakamoto et al. 2005) and their afterglows similar to those of CGRBs provide strong evidence, however, that XRRs/XRFs represent a softer region of

* Tables 1 and 2 are only available in electronic form at the CDS via anonymous ftp to cdsarc.u-strasbg.fr $(130.79 .128 .5)$ or via http://cdsweb.u-strasbg.fr/cgi-bin/qcat?]/A+A/508/593 a continuous GRB distribution (e.g., Lamb et al. 2005; Sakamoto et al. 2008).

A unified picture of the subclasses of GRBs can be obtained by attributing the observed differences in their peak energy to the same objects being observed at different angles with respect to the symmetry axis of the GRB jet (e.g., Yamazaki et al. 2002). The kinetic energy in the jet per solid angle $\varepsilon$ is usually parametrized as a top hat (e.g., Rhoads 1999; Woods \& Loeb 1999), Gaussian (Zhang \& Mészáros 2002), power-law structured outflow with $\varepsilon \propto\left(\theta / \theta_{\text {jet }}\right)^{-q}$ (Mészáros et al. 1998), or a top hat with lower energetic wings. The resulting shape of the afterglow light curve then depends on the viewing angle and jet structure (e.g., Rossi et al. 2002).

In an inhomogeneous or structured jet model, the initial bulk Lorentz factor as well as the specific deceleration time and radius are dependent on the distance to the symmetry axis of the jet (Kumar \& Granot 2003). Hence, a geometric offset in the observers' line of sight from the jets' symmetry axis will have a distinct signature in the optical light curve (e.g., Granot \& Kumar 2003). Because of the relativistic beaming of the decelerating ejecta, an observer located off-axis to the central jet will see a rising optical afterglow light curve at early times (e.g., Panaitescu et al. 1998; Granot et al. 2002). The steepness of the rise would then be characteristic of the off-axis angle and the 
jet structure: the farther the observer is located from the central emitting cone or the faster the energy per solid angle decreases outside the central jet, the shallower is the observed rise in a structured jet model (Panaitescu \& Vestrand 2008). A rest-frame peak energy $E_{\text {peak }}^{\text {rest }}$ of the spectrum of the prompt phase consistent with an XRF would thus correspond to a shallow rise or early plateau phase of the afterglow. With decreasing off-axis angle, both $E_{\text {peak }}^{\text {rest }}$ and the optical afterglow rise index will increase to resemble those of XRRs and steeper rising early afterglow light curves.

\section{Observations}

At $T_{0}=07: 13: 10$ UT on 10 July 2008, Swift triggered and located GRB 080710, but did not slew immediately to the burst (Sbarufatti et al. 2008). Because of an observing constraint, observations with the two narrow field instruments, the X-ray(XRT; Burrows et al. 2005) and UV/Optical Telescope (UVOT; Roming et al. 2005) started $0.87 \mathrm{~h}$ and $0.89 \mathrm{~h}$ after the trigger (Landsman \& Sbarufatti 2008). The burst had a relatively smooth fast rise - exponential decay temporal structure with $T_{90}=120 \pm 17 \mathrm{~s}$ in the $15-350 \mathrm{keV}$ band and weak indication of a precursor $120 \mathrm{~s}$ before the main peak (Tueller et al. 2008). Above $100 \mathrm{keV}$, the burst was only marginally detected by BAT and its spectrum is well described with a single power law of index $-1.47 \pm 0.23$ and a total fluence in the $15-150 \mathrm{keV}$ range of $(1.4 \pm 0.2) \times 10^{-6} \mathrm{erg} / \mathrm{cm}^{2}$ (Tueller et al. 2008). Using the spectral slope from the BAT data, and following Sakamoto et al. (2009), the peak energy of the prompt emission spectrum can be constrained to be $110_{-60}^{+340} \mathrm{keV}$, including the uncertainties in the BAT power law slope. The fluence ratio of GRB 080710 between the two BAT bands $25-50 \mathrm{keV}$ and $50-100 \mathrm{keV}$ is $S$ (25$50 \mathrm{keV}) / S(50-100 \mathrm{keV})=0.70 \pm 0.15$, and the burst thus qualifies as a CGRB in the observers' frame, with errors ranging to a fluence ratio similar to those of XRRs when applying the working definition of Sakamoto et al. (2008).

Assuming a spectral shape of a Band function (Band et al. 1993 ) with a peak energy of around $110 \mathrm{keV}$ and a high energy index of -2.5 , standard $\Lambda$ CDM cosmology $\left(\Omega_{\mathrm{M}}=0.27\right.$, $\left.\Omega_{\Lambda}=0.73, H_{0}=71 \mathrm{~km} \mathrm{~s}^{-1} \mathrm{Mpc}^{-1}\right)$ and a redshift $z$ of 0.845 (Perley et al. 2008; Fynbo et al. 2009), we derive a bolometric ( $1 \mathrm{keV}$ to $10 \mathrm{MeV}$ ) energy release for GRB 080710 of $\log E_{\gamma, \text { iso }}[\mathrm{erg}]=51.75$ with a rest-frame peak energy of $E_{\text {peak }}^{\text {rest }} \sim$ $200 \mathrm{keV}$. Peak energies of the observed prompt spectrum of $50 \mathrm{keV}, 300 \mathrm{keV}$, or $500 \mathrm{keV}$ result in $\log E_{\gamma \text {,iso }}[\mathrm{erg}] \approx 51.70$, 51.94 , or 52.14, respectively. Compared to a sample of previous bursts of known redshift (e.g., Amati et al. 2008), these estimates place GRB 080710 at the lower energy end of GRBs, with an inferred bolometric energy release of around $10^{3}$ times less than the extremely energetic GRB 080916C (Abdo et al. 2009; Greiner et al. 2009a). Hence, a low $E_{\text {peak }}^{\text {rest }}$ in the $50-200 \mathrm{keV}$ range is also supported by the Amati relation (Amati et al. 2002), and is consistent with the most reliable estimate derived using the BAT spectral slope. Given the low redshift and prompt emission properties of GRB 080710 , it thus seems very likely that $E_{\text {peak }}^{\text {rest }}$ is in a range that is typically associated with a XRR in the GRB rest frame (100-300 keV, Sakamoto et al. 2008), although a hard burst cannot be completely excluded by the observations.

GROND (Greiner et al. 2008) at the $2.2 \mathrm{~m} \mathrm{MPI/ESO} \mathrm{tele-}$ scope at LaSilla observatory responded to the Swift trigger and initiated automated observations, which started $384 \mathrm{~s}$ after the burst. During the first two hours, only the $g^{\prime} r^{\prime} i^{\prime} z^{\prime}$ CCDs of GROND were operating. Observations in all seven colors $g^{\prime} r^{\prime} i^{\prime} z^{\prime} J H K_{\mathrm{S}}$ simultaneously started $1.98 \mathrm{~h}$ later and continued until the start of the local nautical twilight at 10:27 UT. Afterwards, GROND switched to a NIR-only mode, where only imaging in $J H K_{\mathrm{S}}$ was performed. TLS imaging was obtained between 00:09 UT and 01:43 UT on 11 July 2008 in filters $B V R$ and $I$ (Schulze et al. 2008). In addition, GROND imaged the field of GRB 080710, both 3 and 4 days after the burst. A finding chart of the field of GRB 080710 is shown in Fig. 1.

The XRT light curve was downloaded from the XRT lightcurve repository (Evans et al. 2007), and spectra were obtained with the xrtpipeline tool using the latest calibration frames from the Swift CALDB and standard parameters. The spectra were fitted using the XSPEC package (Arnaud 1996) with a foreground hydrogen column density at the Galactic value of $N_{\mathrm{H}}=4.1 \times 10^{20} \mathrm{~cm}^{-2}$ (Kalberla et al. 2005). Optical/NIR data (see Tables 1 and 2) were reduced using standard IRAF tasks (Tody 1993) similar to the procedure outlined in Krühler et al. (2008).

\section{Results}

\subsection{Afterglow light curve}

The optical light curve (Fig. 2) exhibited two salient features during observations. First, it shows an initial rise in brightness to a peak at around $2000 \mathrm{~s}$, and second, there is a break in the light curve at roughly $10 \mathrm{ks}$.

The light curve was parametrized with an empirical model of three smoothly connected power laws. The global $\chi^{2}$ of $F_{v, i}(t)$, where $i$ denotes the individual filter or bandpass, was minimized by assuming an achromatic functional form of $F_{v, i}(t)=\eta_{v, i} \times$ $F_{v}(t)$, where only the overall flux normalization $\eta_{v, i}$ depends on the filter, and $F_{v}(t)$ was adapted from Liang et al. (2008). As a result of the high precision of the data and good sampling in the time domain, all parameters were allowed to vary and are presented in Table 3. In principle, all fit parameters depend on the choice of $T_{0}$. Setting $T_{0}$ to the time of the precursor (i.e., $-120 \mathrm{~s})$, we find that the fit parameters describing the early/late power laws vary by a maximum of $20 \%$ and $2 \%$, respectively. Hence, the uncertainty in $T_{0}$ does not change the results derived significantly or affect the overall conclusions.

The decay after the peak at $2 \mathrm{ks}$ with an index of $-0.63 \pm 0.02$ is too shallow to be explained by the normal decay phase, and the late temporal slope of $-1.57 \pm 0.01$ is roughly consistent with the closure relations for the normal decay in the $v_{\mathrm{m}}<v<v_{\mathrm{c}}$ regime for a homogeneous ISM-type circumburst medium in the slow cooling case ( $\alpha=3 \beta / 2$ e.g., Zhang \& Mészáros 2004). There is therefore no apparent evidence of a jet-break before $350 \mathrm{ks}$, and thus $\theta_{\text {jet }}>10^{\circ}$ according to Sari et al. (1999).

\subsection{Broad-band spectrum}

Using the optical/NIR and X-ray data, the afterglow spectrum can be constrained over a broad wavelength range. Four different time intervals were selected to construct a broad-band spectral energy distribution (SED, Fig. 3). The different epochs are indicated in the light curve plot with shaded regions, and the SED fit parameters are presented in Table 4.

As already indicated by the light curve, there is no sign of spectral evolution throughout the observation. Both the early turnover from rising to falling, as well as the second break are achromatic with high measurement accuracy. The optical/NIR SED alone is consistent with a power law of the X-ray spectral index without strong signatures of curvature due to intrinsic 
Table 3. Light curve fits.

\begin{tabular}{|c|c|c|c|c|c|c|c|c|c|}
\hline Bands & $F_{v}(t)$ & $\alpha_{\mathrm{r}}^{a}$ & $s_{1}$ & $t_{\mathrm{b}, 1}[\mathrm{~s}]$ & $\alpha_{d 1}^{a}$ & $s_{2}$ & $t_{\mathrm{b}, 2}[\mathrm{~s}]$ & $\alpha_{\mathrm{d} 2}^{a}$ & $\chi^{2} /$ d.o.f. \\
\hline$\overline{g^{\prime}}$ & $\mathrm{TPL}^{(b)}$ & $1.20 \pm 0.11$ & $2.2 \pm 0.5$ & $1775 \pm 62$ & $-0.64 \pm 0.04$ & $7.2 \pm 1.7$ & $9665 \pm 170$ & $-1.58 \pm 0.01$ & $58 / 59$ \\
\hline$r^{\prime}$ & $\mathrm{TPL}^{(b)}$ & $1.11 \pm 0.07$ & $2.6 \pm 0.4$ & $1816 \pm 39$ & $-0.65 \pm 0.03$ & $6.7 \pm 1.3$ & $9767 \pm 157$ & $-1.55 \pm 0.01$ & $49 / 60$ \\
\hline$i^{\prime}$ & $\mathrm{TPL}^{(b)}$ & $1.10 \pm 0.05$ & $3.1 \pm 0.5$ & $1836 \pm 37$ & $-0.63 \pm 0.03$ & $5.5 \pm 1.2$ & $9752 \pm 185$ & $-1.56 \pm 0.02$ & $52 / 60$ \\
\hline$z^{\prime}$ & $\mathrm{TPL}^{(b)}$ & $1.10 \pm 0.06$ & $3.4 \pm 0.5$ & $1835 \pm 34$ & $-0.60 \pm 0.04$ & $4.2 \pm 1.0$ & $9795 \pm 268$ & $-1.56 \pm 0.03$ & $61 / 59$ \\
\hline$J H K$ & $\mathrm{DPL}^{(c)}$ & - & - & - & $-0.53 \pm 0.15$ & $5.7 \pm 1.7$ & $9542 \pm 527$ & $-1.57 \pm 0.15$ & $84 / 99$ \\
\hline$g^{\prime} r^{\prime} i^{\prime} z^{\prime} J H K_{\mathrm{S}}$ & $\mathrm{TPL}^{(b)}$ & $1.11 \pm 0.03$ & $2.9 \pm 0.2$ & $1829 \pm 19$ & $-0.63 \pm 0.02$ & $5.7 \pm 0.5$ & $9763 \pm 83$ & $-1.57 \pm 0.01$ & $425 / 362$ \\
\hline$g^{\prime} r^{\prime} i^{\prime} z^{\prime} J H K_{\mathrm{S}}+\mathrm{X}$-ray & $\mathrm{TPL}^{(b)}$ & $1.11 \pm 0.03$ & $2.9 \pm 0.2$ & $1829 \pm 19$ & $-0.63 \pm 0.02$ & $5.7 \pm 0.5$ & $9759 \pm 82$ & $-1.57 \pm 0.01$ & $488 / 428$ \\
\hline
\end{tabular}

${ }^{a}$ Power law indices $\alpha$ of the segmented light curve, which are connected via breaks with smoothness s at break times $t_{\mathrm{b}}$.

${ }^{b}$ Smoothly connected triple power law.

${ }^{c}$ Smoothly connected double power law.

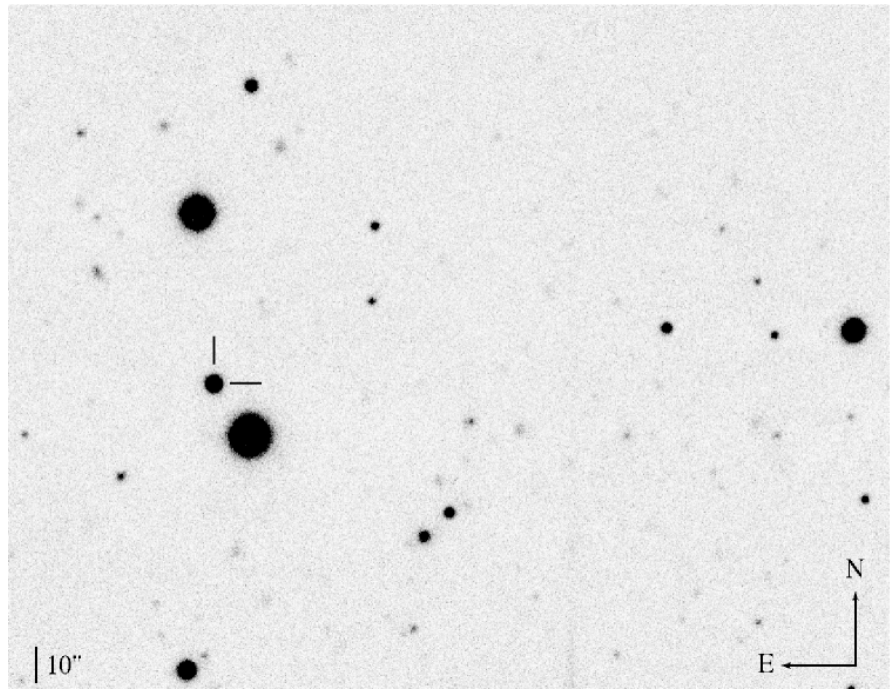

Fig. 1. GROND $r^{\prime}$-band image of the field of GRB 080710 obtained approximately $2 \mathrm{ks}$ after $T_{0}$. The optical afterglow is marked with two lines and the shown image is roughly $4.2^{\prime}$ by $3.2^{\prime}$.

reddening. The expected Galactic foreground extinction $A_{\mathrm{V}}=$ 0.23 mag (Schlegel et al. 1998) however is significant, so some amount of host extinction might be masked by the uncertainty in the foreground correction. In addition, the optical data obtained hardly probe the rest-frame UV regime, where most of any intrinsic extinction would be apparent.

Given that the light curve evolution is similar in both energy ranges and the extrapolation of the X-ray data reproduces the optical flux well, i.e., $\beta_{\text {opt }} \sim \beta_{\text {ox }} \sim \beta_{\mathrm{x}}$, both the optical/NIR and $\mathrm{X}$-ray emission probe the same segment of the afterglow synchrotron spectrum. This implies that the X-ray and optical data are above the typical synchrotron frequency $v_{\mathrm{m}}$ and in the spectral regime of $\max \left(v_{\mathrm{m}}, v_{\mathrm{c}}\right)<v_{\mathrm{opt}}<v_{\mathrm{X}}$, or $v_{\mathrm{m}}<v_{\mathrm{opt}}<v_{\mathrm{X}}<v_{\mathrm{c}}$, where the latter is consistent with a fireball model in a homogeneous ISM and slow cooling case. The spectral index of the electron distribution $p$ would then be $p=2 \beta=2.00 \pm 0.02$ or $2 \beta+1=3.00 \pm 0.02$, respectively. Given that not all bursts are consistent with the closure relations in the basic fireball scenario (e.g., Evans et al. 2008), we consider both cases in the following. Consequentially, the expected break in the synchrotron afterglow spectrum at the cooling frequency $v_{\mathrm{c}}$ could be below the optical at the start of the observations 6 minutes after the burst, or, assuming $v_{\mathrm{m}}<v<v_{\mathrm{c}}$, above the X-rays for the entire observational period.
Table 4. SED fits.

\begin{tabular}{cccc}
\hline \hline Epoch & Spectral index $\beta$ & $N_{\mathrm{H}}^{a}\left[10^{22} \mathrm{~cm}^{2}\right]$ & $\chi^{2} /$ d.o.f. \\
\hline I & $1.00 \pm 0.01$ & $0.19 \pm 0.09$ & $36 / 36$ \\
II & $0.99 \pm 0.01$ & $0.17 \pm 0.10$ & $15 / 15$ \\
III & $1.01 \pm 0.01$ & $0.13_{-0.13}^{+0.15}$ & $18 / 19$ \\
IV & $1.01 \pm 0.01$ & $0.53_{-0.53}^{+1.30}$ & $0.3 / 3$ \\
\hline
\end{tabular}

${ }^{a}$ Intrisic hydrogen column desity, in excess of the frozen Galactic foreground of $N_{\mathrm{H}}=4.1 \times 10^{20} \mathrm{~cm}^{-2}$.

\section{Discussion}

A number of previous bursts have shown a rising optical afterglow at early times, e.g., GRBs 060418, 060607A (Molinari et al. 2007) amongst others (e.g., Covino et al. 2008; Krühler et al. 2008; Ferrero et al. 2009; Greiner et al. 2009b; Oates et al. 2009; Rykoff et al. 2009; Klotz et al. 2009). Similar to the X-ray Flash 071031 (Krühler et al. 2009), the optical SED does not show significant evolution during the rise, and all bands peak at the same time.

An achromatic rising component is generally related to either the onset of the fireball forward shock (e.g., Sari et al. 1999) seen face-on, or to an outflow seen off-axis (e.g., Panaitescu et al. 1998). In the first case, the apparent increase in brightness is caused by the increasing number of radiating electrons. The time of the light curve peak at $T_{0}+2 \mathrm{ks}$ is much later than the end of significant $\gamma$-ray emission $\left(T_{0}+40 \mathrm{~s}\right)$, so the afterglow can be described in the thin shell approximation. The jet is then expected to produce a rising early light curve with a peak when the swept-up circumburst medium starts to decelerate the ejecta efficiently. Depending on the profile of the circumburst medium, the rise has indices of $\sim 2\left(v_{\mathrm{c}}<v_{\mathrm{opt}}\right)$ or $3\left(v_{\mathrm{c}}>v_{\mathrm{opt}}\right)$ in an ISM, or $\sim 0.5$ in an wind-like environment (Panaitescu \& Vestrand 2008). Given that the majority of bursts prefer a circumburst medium with an ISM profile, and the late afterglow decline is consistent with this, we thus consider only the ISM, thin shell case in the following.

In the off-axis case, the peak is a geometric effect: as the shock decelerates, the relativistically beamed emission cone widens and gradually enters the sight line of the observer. The light curve morphology is then dependent on the jet structure and off-axis angle $\theta_{\mathrm{obs}}$, and reaches a maximum when $\Gamma \sim$ $\left(\theta_{\mathrm{obs}}-\theta_{\mathrm{c}}\right)^{-1}$, where $\theta_{\mathrm{c}}$ is the angle of an uniform cone around the symmetry axis of the jet.

There is no evidence of chromatic evolution, which would be the case if the peak was caused by a $v_{\mathrm{m}}$ moving through the 


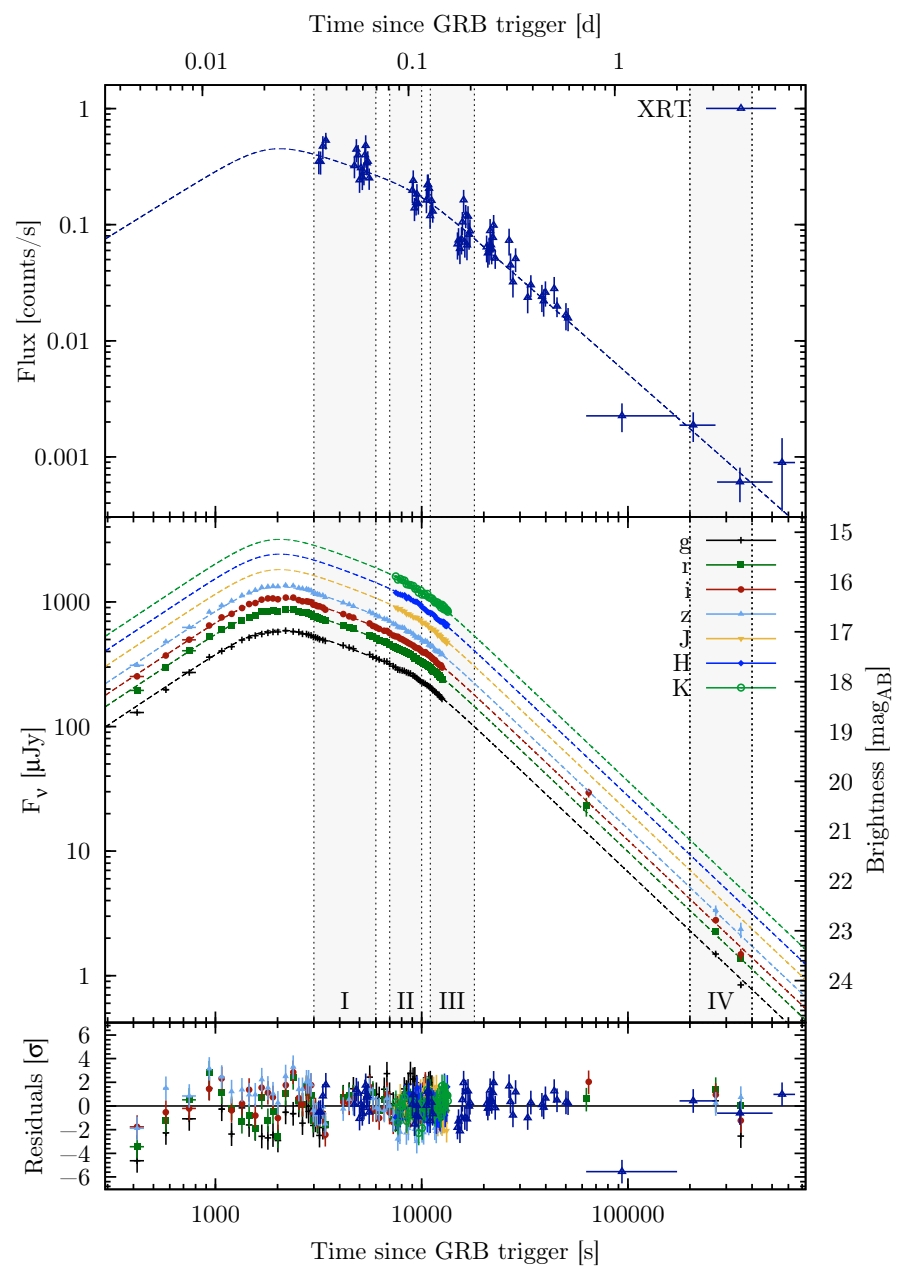

Fig. 2. Light curves of the X-ray (top panel) and optical/NIR (middle panel) afterglow of GRB 080710. Residuals to the combined light curve fit are shown in the lowest panel. Data shown are not corrected for Galactic foreground reddening. Upper limits are not shown to enhance clarity.

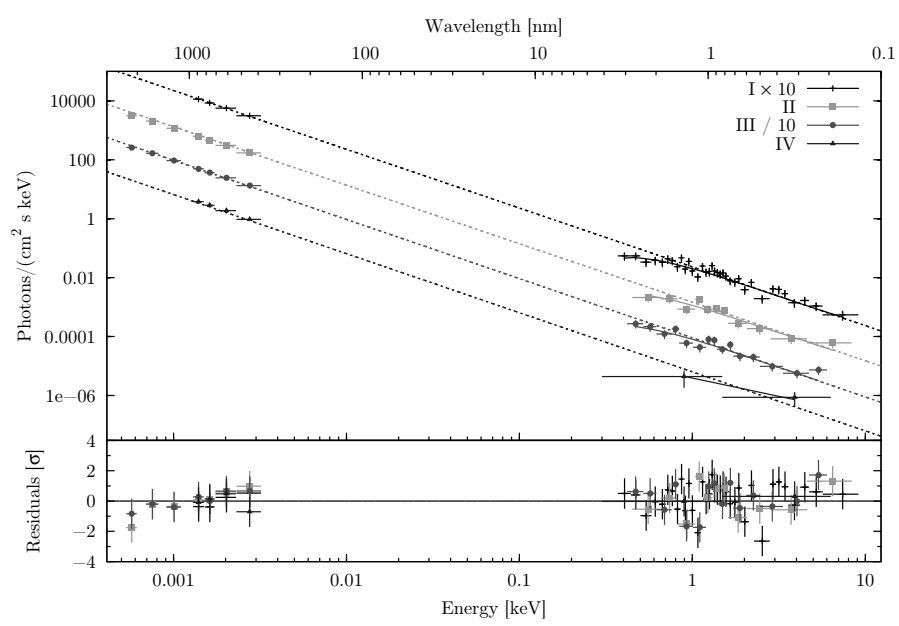

Fig. 3. Broad-band spectral energy distribution from XRT and GROND at different epochs (upper panel). The data were fitted with a powerlaw, modified by a Galactic and intrinsic hydrogen column. The best-fit power law is shown in dotted lines, the best-fit model including the soft $\mathrm{X}$-ray absorption in solid lines. In the lower panel the residuals of the data to the best-fit model. optical bands, or dust destruction, and none of these processes can produce the early achromatic rise. In addition, there is also no sign of a reverse shock, which is expected to decline with a temporal index of -1.75 for $p=2$, or -2.5 for $p=3$. The latter, however, might be masked by a dominating forward shock, or have happened before the start of the GROND observations.

\subsection{On-axis jet in its pre-deceleration phase}

If the light-curve peak was caused by a jet in its pre-deceleration phase, conclusions about the motion of the ultra-relativistic outflow from the central engine can be drawn. Using the time of the absolute light curve maximum $t_{\max } \approx 2 \mathrm{ks}, \log E_{\gamma, \text { iso }}[\mathrm{erg}]=$ 51.70-52.14 and following Molinari et al. (2007), we find initial Lorentz factors of the bulk outflow of around $\Gamma_{0}^{\text {ISM }} \approx 90-100$ ( $\Gamma_{0}^{\text {wind }} \approx 30-40$ ). This is at the very low end of the theoretically expected velocity of the outflow to produce $\gamma$-rays (e.g., Piran 2005), and together with the divergence in the measured (1.1) and expected $(\sim 2-3)$ rise index, makes the scenario of a single on-axis decelerating jet appear somewhat contrived. In addition, a small population of afterglows shows a very late peak or long plateau (e.g., XRF 030723, Fynbo et al. 2004; or GRB 060614 Della Valle et al. 2006), where the derived Lorentz-factor in an on-axis configuration from the optical afterglow peak are uncomfortably small. Furthermore, all previously observed rise indices have a broad distribution (e.g. Panaitescu \& Vestrand 2008; Oates et al. 2009; Rykoff et al. 2009; Klotz et al. 2009, and references therein) from early plateau to very fast rising curves, and they do not cluster around the expected $t^{2-3}$. Consequently, it seems plausible that at least some rising afterglows are not caused by the onset of the afterglow, but rather by a geometrical offset of the observers' sight line with respect to the jets' symmetry axis.

\subsection{Jet seen off-axis}

In contrast to the model of an on-axis jet in its pre-deceleration phase, an off-axis scenario is able to account for a broad range of observed rise indices. The peak time and rise index then relates to the off-axis angle or jet structure and could therefore describe a wide diversity of early afterglows in a single framework (Panaitescu \& Vestrand 2008).

If the energy in the jet outer wings decreases rapidly, the early emission of the line-of-sight ejecta is negligible compared to the central part, and the jet structure can be approximated by a homogeneous top-hat, where the burst energetics can be used to constrain the offset angle. Following Granot et al. (2002), a homogeneous jet with a half opening angle $\theta_{\text {jet }}$ and a Lorentz factor $\Gamma$ seen off-axis at an angle $\theta_{\text {obs }}$ will appear less energetic by a factor of $b^{6}$, where $b=\Gamma\left(\theta_{\mathrm{obs}}-\theta_{\text {jet }}\right)$. Assuming a mean value of $\log E_{\gamma, \text { iso }}[\mathrm{erg}]=53$ and, hence adopting $b^{6} \lesssim 10$ for GRB 080710 , it follows that $\theta_{\text {obs }}-\theta_{\text {jet }} \lesssim 3^{\circ} / \Gamma_{100}$. If viewed onaxis, $E_{\text {peak }}$ would then be $b^{2} E_{\text {peak }}^{\text {obs }} \approx 300 \mathrm{keV}$.

However, the jet geometry need not necessarily be a simple top-hat. In a realistic jet model, the jet viewed off-axis is inhomogeneous, has a top-hat structure with wings of lower energy, or is Gaussian shaped (e.g. Zhang et al. 2003; Eichler \& Granot 2006). In addition, some bursts show evidence that their jet structure consists of two jets (e.g., Berger et al. 2003; Granot et al. 2006; Racusin et al. 2008). In this two-component jet model, a narrow, fast jet produces the prompt $\gamma$-rays and early afterglow, and a slow wide jet dominates the late afterglow emission (Peng et al. 2005). 


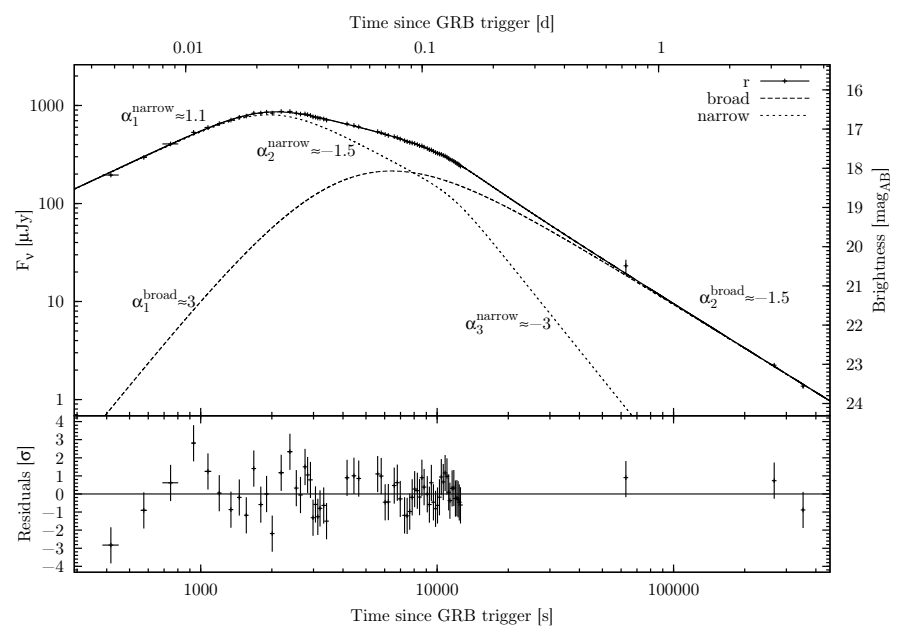

Fig. 4. Tentative two-component fit for GRB 080710 as the superposition of the afterglow of two jets with $v_{\mathrm{m}}<v_{\mathrm{opt}}<v_{\mathrm{X}}<v_{\mathrm{c}}$ and $p \sim 3$ for both components. The narrow jet is viewed slightly off-axis and produces a shallow rise as its emission spreads during deceleration due to relativistic beaming effects. The broad jet is viewed on-axis with $\Gamma_{0} \sim 50, \theta_{\text {jet }}>10^{\circ}$ and has the expected steep rise during its predeceleration phase. Shown is the GROND $r^{\prime}$ band data, all other bands are omitted to enhance clarity.

In these cases, the resulting afterglow light curve in an offaxis geometry is a superposition of two different components: the emission from the ejecta with lower Lorentz factors, which typically dominates at late times, and the relativistic spreading of the decelerating jet around the symmetry axis. The relative energies, jet structure, and offset angle then define the lightcurve morphology. In particular, the delayed onset of the broad jet emission in its pre-deceleration phase might be responsible for the shallow decay observed after the peak. Remarkably, the light curve is equally well ( $\chi^{2}=485$ for 425 d.o.f.) reproduced by the sum of the afterglow of two jets, where the narrow one is viewed slightly off-axis (Fig. 4). Hence, the shallow decay phase could be the result of the superposition of the narrow-jet afterglow and the rise of the broad jet with $\Gamma_{0} \sim 50, \theta_{\text {jet }}>10^{\circ}$ in its pre-deceleration phase. After the emergence of the broad jet afterglow, it subsequently dominates the light curve morphology (Fig. 4). The two-component model thus provides a phenomenological explanation of the shallow decay phase by attributing the shallow slope to the increasing energy dissipation in the predeceleration phase of the broader jet in a specific jet configuration. The opening angle of the narrow jet can be constrained from the light-curve fitting to around $2^{\circ}-4^{\circ}$, but its evolution is masked by the brighter broad jet at later times (Fig. 4). An alternative, jet geometry independent mechanism of energy injection during a decay phase that is shallower than expected, is the refreshed shock scenario (e.g. Rees \& Meszaros 1998; Zhang et al. 2006). A long-lived central engine or a simultaneous ejection of shells with a distribution of Lorentz factors which progressively pile up onto the forward shock could cause the continuous energy injection required for a shallow decay (e.g. Nousek et al. 2006).

An off-axis viewing angle in a two-component or structured jet model with an energy injection can thus provide a consistent picture for the light-curve morphology and the relatively low estimates of $E_{\gamma}$, iso and $E_{\text {peak }}^{\text {rest }}$ of the prompt emission of GRB 080710. In an off-axis scenario, a lower $E_{\text {peak }}^{\text {rest }}$ of the prompt emission spectrum would correspond to a later and fainter afterglow maximum, since both are caused by geometric effects. We caution, that the spectral properties of BAT bursts are generally not well constrained, and GRB 080710 is no exception in this respect. The BAT data, however, indicate a mildly soft event, which could be associated with a XRR in the burst restframe, consistent with the off-axis interpretation of the optical light curve in a unified model.

\section{Conclusions}

The broad-band light curve of the afterglow of GRB 080710 shows two salient features, both achromatic with high precision: an early rise in its brightness, peaking at $\sim 2 \mathrm{ks}$, and a turnover from a shallow to steep decline at $\sim 10 \mathrm{ks}$. The early rise could be caused by a jet in its pre-deceleration phase, or a viewing angle outside the central cone. The latter scenario is naturally able to explain a late-rising afterglow for a soft and weak burst due to a viewing angle offset with respect to the symmetry axis of the jet. An off-axis scenario provides a consistent description of the properties of GRB 080710, and can additionally account for a broad range of rise indices. Consequently, some of the rising afterglow light curves, especially late and shallow ones, might not represent the same class of afterglows that rise because of increasing emission in the pre-deceleration phase, but rather provide evidence of an off-axis location of the observer. The achromatic early increase in brightness observed in the mildly soft GRB 080710 is too shallow to be accounted for with the onset of the afterglow, but significantly steeper than observed in the XRFs 071031 (Krühler et al. 2009) and 080330 (Guidorzi et al. 2009). This might already reflect a common dependence of both $E_{\text {peak }}^{\text {rest }}$, and the rise index of the early optical light curve on the off-axis angle in a unified model: the softer the prompt emission, the more off-axis, and the shallower the rise. This interpretation remains to be tested by the study of a larger sample of early afterglows with well constrained light curves and energetics of the prompt emission from combined Swift/BAT and Fermi/GBM detections, whether and how the structure of an early rise in the optical afterglow is related to prompt emission properties, and in particular, the rest frame $E_{\text {peak }}^{\text {rest }}$ and $E_{\gamma, \text { iso. }}$. A possible correlation would then shed light on the nature of the early afterglow rise, the shallow decay segment, and the jet structure in general.

Acknowledgements. We thank the referee for very helpful comments, which helped to increase the quality of the paper significantly. T.K. acknowledges support by the DFG cluster of excellence "Origin and Structure of the Universe". A.R. and S.K. acknowledge support by DFG grant K1 766/11-3. Part of the funding for GROND (both hardware and personnel) was granted from the LeibnizPrize to Prof. G. Hasinger (DFG grant HA 1850/28-1). S.S. acknowledges support by a Grant of Excellence from the Icelandic Research Fund. This work made use of data supplied by the UK Swift Science Data Centre at the University of Leicester.

\section{References}

Abdo, A. A., Ackermann, M., \& Arimoto, M. 2009, Science, 1688 Amati, L., Frontera, F., Tavani, M., et al. 2002, A\&A, 390, 81 Amati, L., Guidorzi, C., Frontera, F., et al. 2008, MNRAS, 391, 577 Arnaud, K. A. 1996, in Astronomical Data Analysis Software and Systems V, ed. G. H. Jacoby, \& J. Barnes, ASPC Ser., 101, 17

Band, D., Matteson, J., Ford, L., et al. 1993, ApJ, 413, 281

Barthelmy, S. D., Barbier, L. M., Cummings, J. R., et al. 2005, Space Sci. Rev., 120,143

Berger, E., Kulkarni, S. R., Pooley, G., et al. 2003, Nature, 426, 154

Burrows, D. N., Hill, J. E., Nousek, J. A., et al. 2005, Space Sci. Rev., 120, 165 Covino, S., D'Avanzo, P., Klotz, A., et al. 2008, MNRAS, 388, 347

Della Valle, M., Chincarini, G., Panagia, N., et al. 2006, Nature, 444, 1050

Eichler, D., \& Granot, J. 2006, ApJ, 641, L5 
Evans, P. A., Beardmore, A. P., Page, K. L., et al. 2007, A\&A, 469, 379 Evans, P. A., Beardmore, A. P., Page, K. L., et al. 2008, [arXiv:0812.3662] Ferrero, P., Klose, S., Kann, D. A., et al. 2009, A\&A, 497, 729 Fynbo, J. P. U., Sollerman, J., Hjorth, J., et al. 2004, ApJ, 609, 962 Fynbo, J. P. U., Jakobsson, P., Prochaska, J. X., et al. 2009, ApJS, 185, 526 Gehrels, N., Chincarini, G., Giommi, P., et al. 2004, ApJ, 611, 1005 Granot, J., \& Kumar, P. 2003, ApJ, 591, 1086

Granot, J., Panaitescu, A., Kumar, P., \& Woosley, S. E. 2002, ApJ, 570, L61 Granot, J., Königl, A., \& Piran, T. 2006, MNRAS, 370, 1946

Greiner, J., Bornemann, W., Clemens, C., et al. 2008, PASP, 120, 405 Greiner, J., Clemens, C., Krühler, T., et al. 2009a, A\&A, 498, 89

Greiner, J., Krühler, T., McBreen, S., et al. 2009b, ApJ, 693, 1912

Guidorzi, C., Clemens, C., Kobayashi, S., et al. 2009, A\&A, 499, 439

Heise, J., in't Zand, J., Kippen, R. M., \& Woods, P. M. 2001, in Gamma-ray Bursts in the Afterglow Era, ed. E. Costa, F. Frontera, \& J. Hjorth, 16

Kalberla, P. M. W., Burton, W. B., Hartmann, D., et al. 2005, A\&A, 440, 775

Kippen, R. M., Woods, P. M., Heise, J., et al. 2003, in Gamma-Ray Burst and Afterglow Astronomy 2001, ed. G. R. Ricker, \& R. K. Vanderspek, AIPC, 662,244

Klotz, A., Boër, M., Atteia, J. L., \& Gendre, B. 2009, AJ, 137, 4100

Krühler, T., Küpcü Yoldaş, A., Greiner, J., et al. 2008, ApJ, 685, 376

Krühler, T., Greiner, J., McBreen, S., et al. 2009, ApJ, 697, 758

Kumar, P., \& Granot, J. 2003, ApJ, 591, 1075

Lamb, D. Q., Donaghy, T. Q., \& Graziani, C. 2005, ApJ, 620, 355

Landsman, W. B., \& Sbarufatti, B. 2008, GCN, 7965

Liang, E.-W., Racusin, J. L., Zhang, B., Zhang, B.-B., \& Burrows, D. N. 2008, ApJ, 675, 528

Mészáros, P., Rees, M. J., \& Wijers, R. A. M. J. 1998, ApJ, 499, 301

Molinari, E., Vergani, S. D., Malesani, D., et al. 2007, A\&A, 469, L13

Nousek, J. A., Kouveliotou, C., Grupe, D., et al. 2006, ApJ, 642, 389
Oates, S. R., Page, M. J., Schady, P., et al. 2009, MNRAS, 395, 490 Panaitescu, A., Mészáros, P., \& Rees, M. J. 1998, ApJ, 503, 314 Panaitescu, A., \& Vestrand, W. T. 2008, MNRAS, 387, 497

Peng, F., Königl, A., \& Granot, J. 2005, ApJ, 626, 966

Perley, D. A., Chornock, R., \& Bloom, J. S. 2008, GCN, 7962

Piran, T. 2005, Rev. Mod. Phys., 76, 1143

Preece, R. D., Briggs, M. S., Mallozzi, R. S., et al. 2000, ApJS, 126, 19 Racusin, J. L., Karpov, S. V., Sokolowski, M., et al. 2008, Nature, 455, 183 Rees, M. J., \& Meszaros, P. 1998, ApJ, 496, L1

Rhoads, J. E. 1999, ApJ, 525, 737

Roming, P. W. A., Kennedy, T. E., Mason, K. O., et al. 2005, Space Sci. Rev., 120, 95

Rossi, E., Lazzati, D., \& Rees, M. J. 2002, MNRAS, 332, 945

Rykoff, E. S., Aharonian, F., Akerlof, C. W., et al. 2009, ApJ, 702, 489

Sakamoto, T., Lamb, D. Q., Kawai, N., et al. 2005, ApJ, 629, 311

Sakamoto, T., Hullinger, D., Sato, G., et al. 2008, ApJ, 679, 570

Sakamoto, T., Sato, G., Barbier, L., et al. 2009, ApJ, 693, 922

Sari, R., Piran, T., \& Halpern, J. P. 1999, ApJ, 519, L17

Sbarufatti, B., Baumgartner, W. H., Evans, P. A., et al. 2008, GCN, 7957

Schlegel, D. J., Finkbeiner, D. P., \& Davis, M. 1998, ApJ, 500, 525

Schulze, S., Kann, D. A., Rossi, A., et al. 2008, GCN, 7972

Tody, D. 1993, in Astronomical Data Analysis Software and Systems II, ed. R. J.

Hanisch, R. J. V. Brissenden, \& J. Barnes, ASPC Ser., 52, 173

Tueller, J., Barthelmy, S. D., Baumgartner, W., et al. 2008, GCN, 7969

Woods, E., \& Loeb, A. 1999, ApJ, 523, 187

Yamazaki, R., Ioka, K., \& Nakamura, T. 2002, ApJ, 571, L31

Zhang, B., \& Mészáros, P. 2002, ApJ, 571, 876

Zhang, B., \& Mészáros, P. 2004, Int. J. Mod. Phys. A, 19, 2385

Zhang, B., Fan, Y. Z., Dyks, J., et al. 2006, ApJ, 642, 354

Zhang, W., Woosley, S. E., \& MacFadyen, A. I. 2003, ApJ, 586, 356 